\title{
Without Heat and Work - Further Remarks on the Gyftopoulos-Beretta Exposition of Thermodynamics
}

\author{
J. Badur ${ }^{1}$, M. Feidt ${ }^{2}$, P. Ziółkowski ${ }^{1}$ * \\ ${ }^{1}$ Energy Conversion Department, Institute of Fluid Flow Machinery, Polish Academy of Sciences, \\ Fiszera 14, 80-231, Gdańsk, Poland \\ E-mail: jb@imp.gda.pl,pziolkowski@imp.gda.pl \\ ${ }^{2}$ University of Lorraine, Laboratory of Energetics \& Theoretical \& Applied Mechanics 2, \\ Avenue de la Forêt de Haye, 54518 Vandœuvre-lès-Nancy, France \\ E-mail: Michel.Feidt@univ-lorraine.fr
}

Received 16 January 2017, Accepted 24 August 2018

\begin{abstract}
Developed during recent two decades the new exposition of thermodynamics developed by Gyfropoulos and Beretta [1] is aimed both to remove logical circularities in teaching as well in removing obstacles for natural generalization of this science. Keeping the line of reasoning, following Gyftopoulos and Beretta, we will introduce the basic concepts of thermodynamics without the notion of "heat" and "work", and will extend the Gyftopoulos and Beretta exposition into three-dimensional continuum thermodynamics [2]. In proposed approach notion of "energy" and "energy interactions" play a dominant role. The main problem connected with the internal energy concept as a form of ,energy storage" and the transformations of different forms of energy are discussed. Balance of energy is finally presented as a sum of internal, kinetic, potential and electromagnetic energies in the system that are compensated by the total energy flux, which consists of work, heat, chemical, electrical, magnetical and radiative energy fluxes at the system boundaries [3]. The law of energy (and mass) conservation can be considered as the most important one, which is superior over any other laws of nature. An example of "neo-classical" Navier-Stokes equation, being a model thermodynamically consistent, is developed and presented in details.
\end{abstract}

Keywords: balance of energy, entropy flux, volume flux, neo-classical Navier-Stokes equation

\section{Introduction}

Thermodynamics is probably the oldest paradigm of science on the Earth, even oldest than astronomy and mathematics. Its main goal is to describe nature phenomena in terms of "peripatetical motion" and its "entelechy" [1]. Nowadays, thermodynamics possesses the same aims but expressed in modern technical language: to describe all known [or yet unknown] phenomena in terms of energy and its possibility for conversion. For example, nowadays, the most related notion to "entelechy" is internal energy.

From the view point of bases of our civilization, it is important that there exist a concept that unifying our understanding of experience [3,4]. But it should be remembered that the popular use of the word "energy" refers to capacity of certain material bodies to perform work or other useful tasks. This capacity to work represents another quantity that scientist call "available energy". If distinct from "energy", the concept of "available energy" is related to both first and second law of thermodynamics. In other words, the concept of energy is the essence only of the first law, and up to date, cannot be involved as a postulate coming from other principles of science, including even Quantum Field Theory, where "energy" is always before "action" [5-15].

Generally, the main aim of this paper is a specific adaptation of the Gyftopoulos-Beretta approach to threedimensional, local presentation of the laws of thermodynamics on the ground of continuum physics. We have shown that reasoning based on global formulation in time and space can be adapted to a local formulation of thermodynamics within the framework of continuum thermodynamics, with some consequences for the co-called Irreversible Thermodynamics and Extended Nonequilibrium Thermodynamics.

\section{Balance of Thermodynamic Parameters}

The fundamental question of thermodynamics is how state parameters [global: $X_{(k)}=\iint_{A} \rho x_{(k)} d A$ or local] evolve during physical processes, for instance, how specific volume and specific entropy change during simple izoenergetetic process in water steam. Stating on the original line of reasoning (Carnot, Rankine, Reech, Clausius, Gibbs) and extending Hatsopoulos, Keenan, Gyftopoulos and Beretta approach, we propose to write following evolution equations $[1,5]$ :

$d_{(k)}^{*} X_{(k)}=\frac{\partial_{(k)}^{*} F_{(k)}}{a_{(k)}}+N_{(k)}, k=1,2,3 ., .$.

Here increment $d_{(k)}^{*} \quad$ means any kind of substantial differentiation in time $(d / d t)$, adapted for type tensor, quaternion, spinor, etc.. In a case of simple conversion, where: $k=1,2 ; \quad X_{(1)} \equiv V, \quad X_{(2)} \equiv S ; \quad a_{(1)} \equiv p, \quad a_{(2)} \equiv \theta$; 
$F_{(1)} \equiv W, F_{(2)} \equiv Q$, Eq. (1) reduce to the volume and entropy evolution equations respectively:

$d V=\frac{\partial^{*} W}{p}+N_{V} d t \quad ; \quad d S=\frac{\partial^{*} Q}{\theta}+N_{S} d t$

Here appears some "fluxes" of volume and entropy but defined, according to original Rankine-Clausius reasoning, through corresponding fluxes of energy. However, looking for more generic quantities, instead of these particular definitions one can define the local volume flux $\vec{h}_{v}$ and local entropy flux $\vec{h}_{s}$ to be:

$$
\frac{\partial^{*} W}{p}=\left[\iint_{A_{w}} \vec{h}_{V} \cdot \vec{n} d A\right] d t ; \quad \frac{\partial^{*} Q}{\theta}=\left[\iint_{A_{q}} \vec{h}_{S} \cdot \vec{n} d A\right] d t
$$

In Eqs. (2) and (3), according to Clausius' pioneering assumption, some uncompensated energy transformations $N_{(k)} ; \quad N_{V}=\iiint_{V} \rho n_{V} d V ; \quad N_{s}=\iiint_{V} \rho n_{S} d V$ are present in theses balances. In practice, the specific densities $n_{V} ; n_{s}$ are treated as irreversible production of a state control variable. However, uncompensated heat transformation $N_{S}$ has been introduced by Clausius in 1854, yet before his own definition of "disaggregation" (1862) and “entropy" (1865) [4]. However, uncompensated work transformation $N_{V}$ has been also introduced for balance of chemical amounts by Duhem [12]. Remembering that $V=\iiint_{V} \rho v d V$ and $S=\iiint_{V} \rho \eta d V$ in local statement, the balances (2) take a final form:

$$
\rho \frac{d}{d t} v=\operatorname{div} \vec{h}_{V}+\rho n_{V} ; \rho \frac{d}{d t} \eta=\operatorname{div} \vec{h}_{s}+\rho n_{s}
$$

It should be added that the thermodynamical volume $v$ is not a simple inverse of density $\rho$. Another then thermodynamical arguments for balance of volume has recently been introduced by $\mathrm{H}$. Brenner [2].

\subsection{Constitution of the Entropy and Volume Flux}

What is a role of the entropy and volume balance [Eq. (2) or Eq. (4)] ? Do and should be treated as equilibrium or non-equilibrium quantities? Why within the local formulations of thermodynamics these balances are omitted? For instance, it is well-known that Navier-Stokes equation, without balances Eqs. (4) $)_{1}$ and $(4)_{2}$ is thermodynamically correct and consistent?

Therefore, keeping the original Sadi Carnot line of reasoning, where balance of volume are explicitly used for calculations, and, additionally, balance of entropy is a main postulate expressed as a "law of caloric conservation", we may assume that state variables $V$ and $S$ of working continuum change both under conversion and external sources of process described by a proper set of equations. From thermodynamical point of view, no details of momentum transport are important but the "evolution" of main intensive state variables. Thus, solving of balances Eqs. (4) $)_{1}$ and (4) $)_{2}$ are only possible if fluxes of appropriate quantities (e.i. local volume flux $\vec{h}_{V}$ and local entropy flux $\vec{h}_{S}$ ) are defined by some constitutive relations.

Both vectors: $\vec{h}_{v}$ and $\vec{h}_{s}$ must have also a contribution to the energy flux vector - taking Eqs. (2) and (3) into account, defining additional mechanical volumetric $\vec{F}_{m}$ and thermal $\vec{F}_{q}$ energy flux to be:

$\vec{F}_{m}=p \vec{h}_{V} \quad$ and $\quad \vec{F}_{q}=\theta \vec{h}_{S}$

Parameters $p$ and $\theta$ should be treated here as some lagrangian multipliers [8]. Proposed here the volume flux of energy $\vec{h}_{v}$ is consistent with a model of diffuse volume transport recently developed by H. Brenner [2]. He defines the volume flux by a kind of volume velocity $\vec{h}_{v}=\vec{v}_{v}$ which is fundamentally different from the mass velocity $\vec{v}$ introduced 260 years back by Euler. Brenner underlines the thermodynamical role of $\vec{v}_{v}$ velocity calling it's "the work velocity". Surprisingly, such extended fluid kinematics with two fluid velocities is independently postulated by the GENERIC (General Equation for Non-Equilibrium Reversible-Irreversible Coupling) - field theoretical treatment of continuum physics, discovered in pioneering paper by Grmela and Öttinger [13].

\subsection{Balance of a Volume Tensor}

The balance of thermodynamical volume eq. (4) 1 cannot be directly treated as another form of balance of mass, since $v \neq \rho^{-1}$. Balance (4) 1 should be interpreted as a balance of state variable for mechanical-type of contribution to the internal energy. In solid working continuum the role of thermodynamic volume plays an elastic tensor of deformation or, more generally, to the symmetric volume tensor $\vec{v}=v_{i j} \vec{e}_{i} \otimes \vec{e}_{j}$. Then, instance of (4) 1 we postulate:

$\rho \frac{d}{d t} \vec{v}=\operatorname{divh}+\rho \vec{n}_{v}$

Physical interpretation of the volume flux $\mathbf{h}=h_{i j k} \vec{e}_{i} \otimes \vec{e}_{j} \otimes \vec{e}_{k}$ is rather complex - in the literature it appears in the case of so-called weekly non-local plasticity [14]. However, the irreversible production of volume tensor $\vec{n}_{V}$ is present in many irreversible solid phenomena (creep deformation tensor, slip and plastic deformation tensor, etc). For instance, heat engines used in aeronautics are based on zirconia solids as a working continuum and the austenite-martensite phase transition as a main form of energy storage. It is observed that even after few thousand cycles, this working continuum degrades so much that it appears of irreversible plastic deformation induced by a cyclic phase transition.

\subsection{Partial Balance of Kinetic Energy}

Recognized by Coriolis (1842) and Umov (1878), actually, we balance the kinetic energy of a simple continuum by using the balance of momentum (Newtonian equation of motion). The scalar property of energy will be related with momentum only if we do scalar multiplication of momentum balance by the specific momentum $\vec{v}$ : 
$\vec{v} \cdot\left[\rho \frac{d}{d t} \vec{v}=\operatorname{div} \vec{t}+\rho \vec{b}\right]$

Assuming, that tensor of momentum flux for simple working fluid is defined as additive contribution of spherical pressure tensor and the viscous laminar fluid $\vec{t}=-\pi \vec{I}+\vec{\tau}$ we can also split contribution of energy into recoverable and dissipative parts:

$\rho \vec{v} \cdot \frac{d}{d t} \vec{v}=\rho \frac{d}{d t} \kappa=\rho \vec{v} \cdot \vec{b}+\rho \pi \frac{d}{d t} \rho^{-1}$

$+\operatorname{tr}(\vec{\tau} \vec{d})-\operatorname{div}(-\pi \vec{v}+\vec{\tau} \vec{v})$

In the above equation we note: the rate of change of kinetic energy depends on gain of production $\rho \vec{v} \cdot \vec{b}$ taken from diminishing gravitational energy, the amount of energy conversion $\rho \pi \frac{d}{d t} \rho^{-1}$ [where the pressure $\pi$ means mechanical total pressure which is measurable via a barometer], part of energy dissipation $\operatorname{tr}(\vec{\tau} \vec{d})$ [where $\vec{d}$ is symmetric part of the velocity gradient], and the exchange of energy by a part of mechanical flux $\vec{F}_{\text {work }}=-\pi \vec{v}+p \vec{h}_{v}+\vec{\tau} \vec{v}$. It means that only in a partial balance of energy we can observe explicitly three categories of energy transformations: production, conversion and dissipation.

\section{Neo-classical Navier-Stokes Equations}

Let us consider as shortly as possible an example of the Gyftopoulos-Beretta exposition of thermodynamics strictly adapted to the classical Navier-Stokes-Fourier equations. It leads to a neo-classical Navier-Stokes model of working fluid which is a simple viscoelastic, "heat" conducting fluid. The recoverable constitutive relations are expressed only by the specific state parameters: velocity $\vec{v}$ (specific density of momentum); specific volume $v$ and specific entropy $\eta$. Specific internal energy is a function of intensive parameters $\varepsilon=\varepsilon(v, \eta)$.

Let us start from the primal energy balance in the form of a global equality:

$\frac{d}{d t}(U+K+\Phi)=\oiint_{\partial V}\left(\vec{F}_{\text {work }}+\vec{F}_{\text {heat }}\right) \cdot \vec{n} d A$

Here, for our system $A$ [e.g. a simply connected body $B$ ], with a total volume $V$ and a boundary $\partial V$, oriented with a normal vector $\vec{n}$, we have denoted internal energy, by $U=\iiint_{V} \rho \varepsilon d V ; \quad$ kinetic energy, by: $K=\iiint_{V} \rho \kappa d V$ potential energy of body forces, by: $\Phi=\iiint_{V} \rho \phi d V$. Eq. (1) expresses, that total change of stored energy of a body $B$ is compensated by energy being supplied (extracted) through the mechanical energy flux: $\vec{F}_{\text {work }}=\vec{t} \vec{v}+p \vec{h}_{v}$ and the thermal energy flux: $\vec{F}_{\text {heat }}=\vec{h}_{S} \theta$. Definitions for these fluxes are fundamental for the primal statement of energy balance in the Carnot sense - they contain total, and not only reversible, momentum flux $\vec{t}$ and total not only diffusive flux of entropy $\vec{h}_{s}$ as well as the volume flux $\vec{h}_{v}$. Even though the mechanical and thermal energy fluxes are applied on disjoint side surfaces $\partial V=A_{\text {heat }} \cup A_{\text {work }}$, there is no mathematical obstacle whatsoever, to employ Stokes' theorem on the divergence. Assuming that: $\frac{d}{d t} \rho+\rho \operatorname{div} \vec{v}=0$ or, in other words - satisfaction of mass balance, Eq. (9) may be rewritten:

$\iiint_{V}[(\varepsilon+\kappa+\phi)(\dot{\rho}+\rho \operatorname{div} \vec{v})+\rho(\dot{\varepsilon}+\dot{\kappa}+\dot{\phi})$

$\left.-\operatorname{div}\left(\vec{t} \vec{v}+\vec{h}_{v} p+\vec{h}_{s} \theta\right)\right] d V=0$

This equation is always true, regardless the set of governing equations (that is, equations for mass, momentum, volume and entropy balances) is satisfied or not. It will now be shown that, if the fields and parameters of state satisfy conditions supposed by governing equations, equation of energy receives a very important form allowing for further restrictions to describing fields - it means that even though the solutions may satisfy conservation equations for mass, momentum, volume and entropy, they need not to satisfy conservation equation for energy, and in consequence lead to generating energy ex nihilo.

In Eq. (10), according to Brenner's [2] concept of volume balance, we introduce the following splitting of the volume flux:

$\vec{h}_{v}=\vec{v}+\vec{h}_{v}^{\prime}$

which is consistent with postulate that the volume flux is identical with the volume velocity: $\vec{h}_{v}=\vec{v}_{v}=\vec{v}+\alpha_{B} \operatorname{grad} p$ [2]. Material derivatives of kinetic and potential energy are $\dot{\kappa}=\dot{\vec{v}} \cdot \vec{v}$ and $\dot{\phi}=-\frac{\partial \phi}{\partial \vec{x}} \cdot \dot{\vec{x}}=-\vec{b} \cdot \vec{v}$ respectively. The following identities also occur: $\operatorname{div}(\vec{t} \vec{v})=(\operatorname{div} \vec{t}) \cdot \vec{v}+\vec{t} \cdot \operatorname{grad} \vec{v} \quad$ and $\operatorname{div}\left(\vec{h}_{S} \theta\right)=\theta \operatorname{div} \vec{h}_{\mathrm{s}}+\vec{h}_{S} \cdot \operatorname{grad} \theta . \quad$ According to the wellaccepted tradition, the velocity gradient will be denoted by $\vec{l}=\operatorname{grad} \vec{v}$ and decomposed into symmetric and antisymmetric parts $\vec{l}=\vec{d}+\vec{w}$. Temperature gradient will be denoted by $\vec{g}_{s}=\operatorname{grad} \theta$ and pressure gradient by $\vec{g}_{V}=\operatorname{grad} p$.

Through addition and subtraction of $\pm \theta\left(\rho \dot{\eta}-\rho n_{s}\right)$ and $\pm p\left(\rho \dot{v}-\rho n_{V}\right)$, the integral of equation (10) becomes:

$$
\begin{gathered}
\iiint_{V}\left[\rho \dot{\varepsilon}-(\operatorname{div} \vec{t}+\rho \vec{b}-\rho \dot{\vec{v}}) \cdot \vec{v}-\left(\operatorname{div} \vec{h}_{S}+\rho n_{S}-\rho \dot{\eta}\right) \theta+\right. \\
\quad-\left(\operatorname{div} \vec{h}_{V}+\rho n_{V}-\rho \dot{v}\right) p-(\varepsilon+\kappa+\phi)(\dot{\rho}+\rho \operatorname{div} \vec{v})+ \\
\left.+\theta\left(\rho \dot{\eta}-\rho n_{S}\right)+p\left(\rho \dot{v}-\rho n_{V}\right)-\vec{h}_{S} \cdot \vec{g}_{S}-\vec{h}_{V}^{\prime} \cdot \vec{g}_{V}-\vec{t}^{\prime} \cdot \vec{l}\right] d V=0
\end{gathered}
$$

Once the fields $\rho, \vec{t}, \vec{v}, \vec{h}_{s}, \eta, \vec{h}_{v}, v$ satisfy balance equations of mass, momentum, moment of momentum $\vec{t}=\vec{t}^{\mathrm{T}}$ as well as volume and entropy, including, that $\vec{t} \cdot \vec{l}=\vec{t} \cdot \vec{d}$ and $\vec{t}^{\prime}=\vec{t}-p \vec{I}$ energy Eq. (12) reduces to:

$$
\begin{gathered}
\iiint_{V}\left[\rho \dot{\varepsilon}-\theta\left(\rho \dot{\eta}-\rho n_{S}\right)-p\left(\rho \dot{v}-\rho n_{V}\right)\right. \\
\left.-\vec{h}_{S} \cdot \vec{g}_{S}-\vec{h}_{V}^{\prime} \cdot \vec{g}_{V}-\vec{t}^{\prime} \cdot \vec{d}\right] d V=0
\end{gathered} .
$$


Even if a thermo-elastic fluid is expressed via scalar state parameters $v, \eta$, what is much simple than in solids, the mechanism of the internal energy storage is more complex, due to multiplicative, not additive, contribution to $\varepsilon=\varepsilon(\eta, v)$. The time material (not the spatial one) rate of internal energy is: $\dot{\varepsilon}=\frac{\partial \varepsilon}{\partial \eta} \dot{\eta}+\frac{\partial \varepsilon}{\partial v} \dot{v}$. Therefore, by taking material derivative of the internal energy, we obtain:

$$
\begin{gathered}
\iiint_{V}\left[\rho\left(\frac{\partial \varepsilon}{\partial v}-p\right) \dot{v}+\rho\left(\frac{\partial \varepsilon}{\partial \eta}-\theta\right) \dot{\eta}+\rho \theta n_{S}+\right. \\
\left.+\rho p n_{V}-\vec{h}_{S} \cdot \vec{g}_{S}-\vec{h}_{V}^{\prime} \cdot \vec{g}_{V}-\vec{t}^{\prime} \cdot \vec{d}\right] d V=0
\end{gathered} \cdot
$$

Working fluid will be thermo-elastic in the Carnot sense if, and only if thermodynamic pressure and temperature are connected with internal energy by equations of state $p=\frac{\partial \varepsilon}{\partial v}, \theta=\frac{\partial \varepsilon}{\partial \eta}$. These two constitutive relations are fundamental for proving that balance of total energy can be fulfilled in any processes governed by balance of mass, momentum, moment of momentum, volume and entropy. In the case of ideal gas, the internal energy depends on two constitutive coefficients $c_{v}$ and $c_{p}$ - the specific heat in constant volume and pressure, respectively. From this pair; $c_{v}, c_{p}$ one can obtain another pair - Carnot: (1824)

$$
\begin{aligned}
& R=c_{p}-c_{v} \text { and Poisson }(1831): \gamma=\frac{c_{p}}{c_{v}}: \\
& \varepsilon(\eta, v)=\frac{1}{\gamma-1}(v)^{1 /(\gamma-1)} \exp \left(\frac{\eta}{c_{v}}\right)
\end{aligned}
$$

which leads to the well-established constitutive equations:

$$
p=v^{-\gamma} \exp \left(\frac{\eta}{c_{v}}\right) ; \quad \theta=\frac{1}{\gamma-1}(v)^{1-\gamma} .
$$

Finally, remaining part of the balance of energy is:

$$
S_{e}=\iiint_{V}\left[\rho \theta n_{S}+\rho p n_{V}-\vec{h}_{S} \cdot \vec{g}_{S}-\vec{h}_{V}^{\prime} \cdot \vec{g}_{V}-\vec{t}^{\prime} \cdot \vec{d}\right] d V=0 .
$$

This expression say that dissipative contribution to energy balance should be self-equilibrated, since dissipation does not mean creation ex nihilo. Eq. (17) can be also interpreted as an "inner dissipative conversion of energy". This mean that mechanical dissipative energy can convert into thermal dissipative energy and reverse, thermal dissipative energy can convert to mechanical dissipative energy. The last statement is rather original in the framework of common understanding of second law of thermodynamic. Thus, in some sense Eq. (17) could be treated to be definitions of irreversible contribution $n_{V}$ (volume production) and $n_{s}$ (entropy production). If these contributions would be nonnegative $n_{v} \geq 0, n_{s} \geq 0$ then remaining parts are also nonnegative $\vec{h}_{S} \cdot \vec{g}_{S} \geq 0 ; \vec{h}_{V}^{\prime} \cdot \vec{g}_{V} \geq 0 ; \vec{t}^{\prime} \cdot \vec{d} \geq 0$.

The condition of self-equilibrated dissipation Eq. (17) can be fulfilled also if we define $n_{v}$ and $n_{S}$ in the form of specific dissipation potential $\varpi$. In fluid, the momentum flux tensor $\vec{t}^{\prime}$ becomes an additive composition of spherical and shape viscous contributions; ea. : $\vec{t}^{\prime}=-(\pi-p) \vec{I}+\vec{\tau}$. In general, the viscous part is traceless: $\operatorname{tr}(\vec{\tau})=0$, and the volume-like viscous part is a spherical one: $(\pi-p) \vec{I}$. Thus, the expression $\vec{t} \cdot \vec{d}$ in Eq. (17) turns into: $(\pi-p) \operatorname{div} \vec{v}+\vec{\tau} \cdot \vec{d}$ and

$$
\theta \rho n_{S}+p \rho n_{V}=\vec{h}_{V}^{\prime} \cdot \vec{g}_{V}+\vec{h}_{S} \cdot \vec{g}_{S}+(\pi-p) \operatorname{div} \vec{v}+\vec{\tau} \cdot \vec{d}
$$

However, the viscous work and heat diffusion properties depend on the following dissipative potential:

$$
\begin{aligned}
& \varpi\left(\vec{g}_{V}, \vec{g}_{S}, \vec{d}\right)= \\
& =\frac{1}{2} k_{\eta} \vec{g}_{S} \cdot \vec{g}_{S}+\frac{1}{2} \alpha_{B} \vec{g}_{V} \cdot \vec{g}_{V}+\mu \vec{d} \cdot \vec{d}+\left(\frac{2}{3} \mu-\lambda^{\prime}\right)(\operatorname{tr} \vec{d})^{2}
\end{aligned}
$$

where $k_{\eta}$ is an entropy diffusion coefficient connected with the Fourier conductivity coefficient $k$ simply as $k_{\eta}=\theta k$ and $\mu$ and $\lambda^{\prime}$ are the Stokes shear and volumetric viscosity coefficients and $\alpha_{B}$ is the Brenner coefficient. Making use of Eq. (10) and the definitions of diffusive fluxes: $\quad \vec{\tau}=\frac{\partial \varpi}{\partial \vec{d}}, \quad \vec{h}_{s}=\frac{\partial \varpi}{\partial \vec{g}_{s}}, \quad \vec{h}_{V}^{\prime}=\frac{\partial \varpi}{\partial \vec{g}_{V}}, \pi-p=\frac{\partial \varpi}{\partial \operatorname{tr} \vec{d}}$ finally we obtain the well-established constitutive equations:

$$
\begin{aligned}
& \vec{h}_{S}=k_{\eta} \vec{g}_{S} \quad, \quad \vec{h}_{V}^{\prime}=\alpha_{B} \vec{g}_{V} ; \\
& \vec{\tau}=2 \mu \vec{d}+\frac{2}{3} \mu(\operatorname{tr} \vec{d}) \vec{I} \quad, \quad \pi-p=\lambda^{\prime} \operatorname{tr} \vec{d} .
\end{aligned}
$$

Additionally, several restrictions are imposed on constitutive coefficients - there are - the Alexis Petit inequalities $c_{p}>c_{v}>0$; the Lamè-Kowalski inequality $k_{\eta}>0$, the Duhem inequalities $\mu>0,2 \mu+3 \lambda^{\prime}>0$ and so on. From positivity of dissipation functional it follows also positivity of the Brenner coefficient $\alpha_{B} \geq 0$ [2].

\section{Conclusions}

Summarizing the consequences of the GyftopoulosBeretta thermodynamics exposition - the presented above mathematical model of continuum - the "neo-classical" Navier-Stokes - has a thermodynamic consistency, what means that unknown fields assigned from a proper set of governing equations must additionally fulfill the condition of not creating energy from nothing [ $S_{e}=0$, Eq. (17)].

Second consequence is: energy conversion within the working fluid is impossible if state parameters are defined by other than Eq. (16) constitutive equations. For multiplicative form of specific energy (like Eq. (15)) is, the energy conversion is possible without additional assumption, however, for an additive expression of internal energy, like in thermo-elastic solids, or thermo-electric fluids, convertibility is assured only by additional crossing term in constitutive relations.

Third consequence is the condition of self-equilibrated dissipation, which follows from condition $S_{e}=0$ and frequently postulated in the literature the principle of mutual "inner conversion" of dissipative energy. Therefore, 
numerous inequalities [like the Drucker-Prager inequality for granular solids] used in the literature have their sources in the first, not in the second, law of thermodynamics. Finally, it means that the classical Clausius-Duhem inequalities have no deeper foundations and nowadays should be treated as historic no right composition of Clausius' global entropy inequality with Duhem's local energy inequality.

Fourth consequence is a new role of vector of entropy flux for which we have proposed an independent constitutive Eq. (20). It is helpful solution, since the seminal question of interrelation between thermal energy flux and entropy flux is now eliminated.

\section{References}

[1] E.P. Gyftopoulos, G.P. Beretta, Thermodynamics, Foundations and Applications, New York, 2nd ed. Dover Pub. Inc. Mineola, 2005.

[2] H. Brenner, "Kinematics of volume transport", Physica A 349, 11-59, 2005.

[3] M. Feidt, Thermodynamique et optimisation énergétique des systemes et procédés, Paris, Technique et Documentation, 1987.

[4] J. Badur, Rozwój pojęcia energii, Gdańsk, Wyd. IMP PAN, 2009.

[5] G.P. Beretta, "Axiomatic definition of entropy for nonequilibrium states", Int. J. of Thermodynamics, 11, 3948, 2008.

[6] E. Zanchini, G.P. Beretta, "Removing heat and conceptual loops from the definition of entropy", Int. J. of Thermodynamics, 13, 67-76, 2010.
[7] E.P. Gyftopoulos, "Fundamentals of analysis of process", Energy Convers. Manag., 36, 1525-1533, 1997.

[8] S. Sieniutycz, Conservation Laws in Variational Thermo-Hydrodynamics, Dordrecht, Kluwer Acad. Press, 1994.

[9] D. Jou, J. Casas Vàzquez and G. Lebon, Extended Irreversible Thermodynamics, Berlin, Springer, 2001.

[10] S. Kjelstrup, D. Bedeaux, Non-equilibrium Thermodynamics of Heterogeneous Systems, Singapore, Word Scientific Pub., 2008.

[11] V.A. Cimmelli, Conceptual analysis of the entropy principle in continuum physics, an overview, [in:] eds M. Pilotelli, J.P. Beretta, Proc. 12th Joint Eur. Thermod. Conf., Brescia, July 1-5, 2013, 208-209, 2013.

[12] P. Duhem, "Recherches sur l'hydrodynamique", Ann. Toulouse (2) 3315-377, 379-431 (1901); 4101-169 (1902), 55-61, 197-255, 353-404 (1903).

[13] M. Grmela, H.C. Öttinger, "Dynamics and thermodynamics of complex fluids. I. development of a general formalism", Phys. Rev., 56, 6620-6632, 1997.

[14] T. Fülöp, P. Ván and A. Csatár, Elasticity, plasticity, rheology and thermal stress - an irreversible thermodynamics theory, [in:] eds M. Pilotelli, J.P. Beretta, Proc. 12th Joint Eur. Thermod. Conf., Brescia, July 1-5, 2013, 525-530, 2013.

[15] A. Bejan, Entropy generation through heat and fluid flow, New York, Wiley, 1982. 